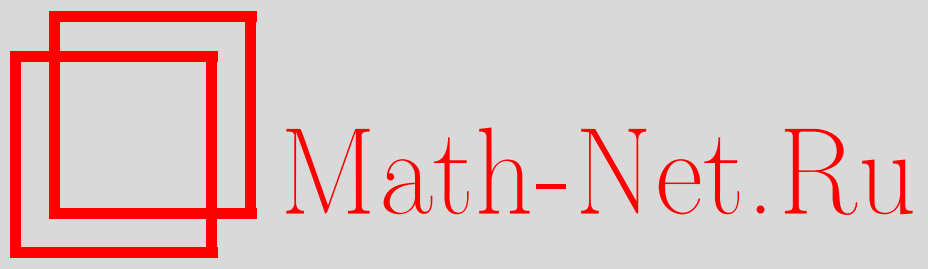

Е. Ю. Бунькова, В. М. Бухштабер, Полиномиальные динамические системы и обыкновенные дифференциальные уравнения, ассоциированные с уравнением теплопроводности, Функи. анализ и его прил., 2012, том 46, выпуск 3, 16-37

DOI: https://doi.org/10.4213/faa3077

Использование Общероссийского математического портала Math$\mathrm{Net.Ru} \mathrm{подразумевает,} \mathrm{что} \mathrm{вы} \mathrm{прочитали} \mathrm{и} \mathrm{согласны} \mathrm{с} \mathrm{пользователь-}$ ским соглашением http://www . mathnet.ru/rus/agreement

Параметры загрузки:

IP : 54.237 .59 .107

26 апреля 2023 г., 09:07:14

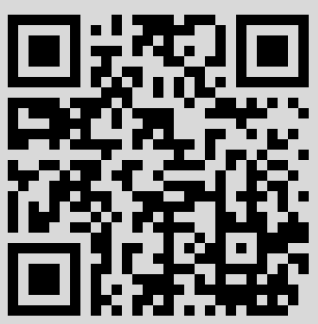




\title{
Полиномиальные динамические системы и обыкновенные дифференциальные уравнения, ассоциированные с уравнением теплопроводности*
}

\author{
(C) 2012. Е. Ю. Бунькова, В. М. БухштаБеР
}

\begin{abstract}
Рассматриваются однородные полиномиальные динамические системы в $n$-мерном пространстве. Каждой такой системе наша конструкция сопоставляет нелинейное обыкновенное дифференциальное уравнение и алгоритм построения решения уравнения теплопроводности. При этом классическое решение, задаваемое функцией Гаусса, соответствует случаю $n=0$, а решения, определенные эллиптической тета-функцией, приводят к уравнению Шази-3 и соответствуют случаю $n=2$. Дается явное описание семейства обыкновенных дифференциальных уравнений, возникающих в нашем подходе, а также связь с широко известными квадратичными динамическими системами Дарбу-Альфана и их обобщениями.
\end{abstract}

\section{$\S 1$. Введение}

Эллиптическая тета-функция $\theta_{1}(z, \tau)$ является решением уравнения теплопроводности. Используя анзац

$$
\phi(z, t)=e^{-\frac{1}{2} h(t) z^{2}+r(t)} \sigma\left(z ; g_{2}(t), g_{3}(t)\right),
$$

где $\phi(z, t)=\theta_{1}(z, 2 \pi i t)$ и $\sigma\left(z ; g_{2}, g_{3}\right)$ - сигма-функция Вейерштрасса с параметрами $g_{2}, g_{3}$, из уравнения теплопроводности

$$
\frac{\partial \psi}{\partial t}=\frac{1}{2} \frac{\partial^{2} \psi}{\partial z^{2}}
$$

мы получаем полиномиальную динамическую систему на функции $g_{2}(t), g_{3}(t)$ и $h(t)$. Решение $h(t)$ этой системы является решением уравнения Шази-3, а функции $g_{2}(t), g_{3}(t)$ являются дифференциальными полиномами от $h(t)$. Таким образом, мы получаем семейство решений уравнения теплопроводности, параметризованное начальными данными задачи Коши для уравнения Шази-3 (см. детали в [1]).

Данная работа посвящена построению и изучению последовательности семейств решений уравнения теплопроводности. Мы получаем семейства решений уравнения теплопроводности, параметризованные полиномиальными динамическими системами в $n$-мерном пространстве, $n=0,1, \ldots$, и начальными данными задачи Коши обыкновенного дифференциального уравнения. В случае $n=2$ мы получаем семейство, включающее семейство, описанное выше.

Содержание работы: В $\S 2$ собраны необходимые факты о классическом одномерном уравнении теплопроводности, в частности, описано действие группы

* Работа выполнена при поддержке грантов РФФИ 11-01-00197-а, 11-01-12067-офи-м-2011, и гранта правительства РФ 2010-220-01-077, договор 11.G34.31.0005. 
$S L(2, \mathbb{C})$ на пространстве решений этого уравнения. В 33 изучается анзац

$$
\psi(z, t)=e^{-\frac{1}{2} h(t) z^{2}+r(t)} \Phi(z ; \boldsymbol{x}(t))
$$

для решений уравнения теплопроводности, где коэффициенты ряда по z функции $\Phi(z ; \boldsymbol{x}(t))$ являются полиномами от $\boldsymbol{x}(t)=\left(x_{2}(t), x_{3}(t), \ldots, x_{n}(t)\right)$. Этот анзац замкнут относительно действия группы $S L(2, \mathbb{C})$. Мы представляем конструкцию, которая сводит задачу решения уравнения теплопроводности в этом анзаце к решению однородной полиномиальной динамической системы. В $\$ 4$ мы сводим однородные полиномиальные динамические системы из предыдущего параграфа к обыкновенным дифференциальным уравнениям специального типа. Таким образом, для любого $n$ мы получаем семейство решений вида $(1.1)$ уравнения теплопроводности. Каждое из этих решений задается конечномерным числовым вектором. В 55 для каждого $n$ мы описываем класс обыкновенных дифференциальных уравнений, полученных в предыдущем параграфе, и связь между этими уравнениями для разных $n$. В $\S 6$ мы изучаем рекуррентные формулы на коэффициенты ряда по $z$ функции $\Phi(z ; \boldsymbol{x}(t))$. В $\S 7$ введена последовательность обыкновенных дифференциальных уравнений порядка $n$, общие решения которых задаются рациональными функциями. Получены условия, необходимые для того, чтобы эти уравнения принадлежали к классу, описанному в $\S 5$. В $\S 8$ мы рассматриваем частные случаи $n=0,1,2,3,4$. В центре нашего внимания дифференциальные уравнения со свойством Пенлеве. В $\S 9$ вводится понятие алгебраически интегрируемой симметричной квадратичной динамической системы, а также более общий анзац для решений уравнения теплопроводности, такой, что каждое решение такой системы в общем положении определяет решение уравнения теплопроводности в этом анзаце.

Авторы благодарны Р. Конту, Ж.-К. Замбрини и Э. Рису за полезные обсуждения результатов работы.

\section{§2. Уравнение теплопроводности}

Уравнение теплопроводности

$$
\frac{\partial \psi}{\partial t}=\frac{1}{2} \frac{\partial^{2} \psi}{\partial z^{2}}
$$

является линейным относительно функции $\psi$ и инвариантным относительно сдвигов аргумента:

- для решений $\psi_{1}$ и $\psi_{2}$ функция $\psi_{1}+\psi_{2}$ также является решением,

- для решения $\psi(z, t)$ функция $\psi\left(z+z_{0}, t+t_{0}\right)$ является решением при любых постоянных $z_{0}$ и $t_{0}$, и, следовательно, функции $\frac{\partial}{\partial z} \psi(z, t)$ и $\frac{\partial}{\partial t} \psi(z, t)$ также являются решениями.

Значит, мы можем сосредоточиться на следующей задаче: найти решения уравнения (2.1), которые в окрестности точки $(0, t)$ задаются рядом

$$
\psi(z, t)=\sum_{k=0}^{\infty} \psi_{k}(t) \frac{z^{k}}{k !} .
$$

В этом случае из уравнения (2.1) следует, что ряд полностью определяется начальными данными $\psi(0, t)=\psi_{0}(t), \psi^{\prime}(0, t)=\psi_{1}(t)$. Более того, для решений вида (2.2) уравнение становится эквивалентным системе $\psi_{k+2}(t)=2 \psi_{k}^{\prime}(t), k=$ $0,1, \ldots$ Следовательно, любое решение вида (2.2) можно представить в виде 
суммы четного решения с начальными условиями $\psi(0, t)=\psi_{0}(t), \psi^{\prime}(0, t)=0$ и нечетного решения с начальными условиями $\psi(0, t)=0, \psi^{\prime}(0, t)=\psi_{1}(t)$.

Рассмотрим функцию вида

$$
\psi(z, t)=e^{-\frac{1}{2} h(t) z^{2}} \phi(z, t),
$$

где $\phi(z, t)$ - полином от $z$.

Лемма 2.1. Функиия $\psi(z, t)$ вида (2.3) является решением уравнения теплопроводности (2.1) тогда и только тогда, когда она является линейной комбинацией с постоянными коэфбициентами функиии Гаусса

$$
\frac{1}{\sqrt{t-c}} \exp \left(\frac{-z^{2}}{2(t-c)}\right)
$$

u ее производных по z. Здесь с-константа.

Доказательство. Пусть $\phi(z, t)$ - полином степени $n$. Положим $\phi(z, t)=$ $\sum_{k=0}^{n} \phi_{k}(t) z^{k}$, где $\phi_{n}(t) \not \equiv 0$. Подставляя $(2.3)$ в $(2.1)$ и деля на $e^{-\frac{1}{2} h(t) z^{2}}$, получаем равенство между двумя полиномами от $z$. В левой части равенства коэффициент при $z^{n+2}$ равен $-\frac{1}{2} h^{\prime} \phi_{n}$, тогда как в правой части он равен $\frac{1}{2} h^{2} \phi_{n}$. Следовательно, $h(t)=\frac{1}{t-c}$ для некоторой постоянной $c$. Теперь в левой части равенства коэффициент при $z^{n}$ равен $\phi_{n}^{\prime}(t)$, тогда как в правой части он равен $-(n+1 / 2) h(t) \phi_{n}(t)$. Следовательно, $\phi_{n}(t)=c_{n}(t-c)^{-n-1 / 2}$ для постоянной $c_{n} \neq 0$. Решением уравнения с таким коэффициентом $\phi_{n}(t)$ является функция $\psi_{n}(z, t)=(-1)^{n} c_{n} \frac{\partial^{n}}{\partial z^{n}}\left(\frac{1}{\sqrt{t-c}} \exp \left(\frac{-z^{2}}{2(t-c)}\right)\right)$. Остальные коэффициенты решения связаны соотношениями

$$
\phi_{k+2}=\frac{2}{(k+2)(k+1)}\left(\phi_{k}^{\prime}(t)+\frac{(2 k+1)}{2} h(t) \phi_{k}(t)\right), \quad k=0,1, \ldots, n-1,
$$

определяющими $\phi_{k}$ однозначно по $\phi_{k+2}$ с точностью до прибавления решения уравнения $\phi_{k}^{\prime}(t)=-\frac{1}{2}(2 k+1) h(t) \phi_{k}(t)$. Таким образом, для полинома $\phi(z, t)$ соответствующим ему решением является сумма функций $\psi_{k}(z, t)$ с постоянными коэффициентам, а функции $\psi_{k}(z, t)$ с точностью до постоянных множителей являются производными функции (2.4).

Группа симметрий. Детальное описание алгебры Ли группы преобразований уравнения теплопроводности и однопараметрических подгрупп, соответствующих базису в этой алгебре Ли, можно найти в [2]. Нам потребуется действие группы $S L(2, \mathbb{C})$ на пространстве решений уравнения теплопроводности.

$$
\begin{aligned}
\text { Пусть } M=\left(\begin{array}{ll}
a & b \\
c & d
\end{array}\right) \in S L(2, \mathbb{C}) \text { и } \\
\Gamma(M) \psi(z, t)=\frac{1}{\sqrt{c t+d}} \exp \left(\frac{-c z^{2}}{2(c t+d)}\right) \psi\left(\frac{z}{c t+d}, \frac{a t+b}{c t+d}\right) .
\end{aligned}
$$

Например, $Г(M) 1$ - классическое решение (2.4) уравнения теплопроводности.

Лемма 2.2. Пусть $\psi(z, t)$ - решение уравнения теплопроводности (2.1). Тогда $\Gamma(M) \psi(z, t)$ - также его решение. Группа $S L(2, \mathbb{C})$ действует на пространстве решений уравнения теплопроводности.

Доказательство получается непосредственной подстановкой (2.5) в (2.1) с использованием соотношения $a d-b c=1$. 


\section{§3. Динамические системы, ассоциированные с уравнением теплопроводности}

Четную (нечетную) регулярную в $z=0$ ненулевую функцию $\psi(z, t)$ можно представить в виде

$$
\psi(z, t)=e^{-\frac{1}{2} h(t) z^{2}+r(t)} z^{2 s} \phi(z, t),
$$

где $s$ - целое неотрицательное число, а четную (нечетную) функцию $\phi(z, t)$ в окрестности точки $z=0$ можно представить в виде ряда

$$
\phi(z, t)=z^{\delta}+\sum_{k \geqslant 2} \phi_{k}(t) \frac{z^{2 k+\delta}}{(2 k+\delta) !},
$$

где $\delta=0$ (соответственно $\delta=1)$. Заметим, что такое представление однозначно; следовательно, для любой функции $\psi(z, t)$ можно говорить о соответствующих функциях $h(t), r(t), \phi(z, t)$. Из $\S 2$ следует, что если функция $\psi$ является решением уравнения теплопроводности, то $s=0$. Далее везде $\delta=0$ или $\delta=1$.

Рассмотрим функцию $\psi(z, t)$ вида $(3.1)$ с $s=0$. Обозначим через $\gamma_{1}(M) h(t)$ и $\gamma_{2}(M) \exp (r(t))$ функции, соответствующие функциям $h(t)$ и $\exp (r(t))$ в представлении вида (3.1) функции $Г(M) \psi(z, t)$.

Лемма 3.1. Имеют место равенства

$$
\begin{aligned}
\gamma_{1}(M) h(t) & =\frac{1}{(c t+d)^{2}} h\left(\frac{a t+b}{c t+d}\right)+\frac{c}{(c t+d)}, \\
\gamma_{2}(M) \exp (r(t)) & =\frac{1}{(c t+d)^{\delta} \sqrt{c t+d}} \exp \left(r\left(\frac{a t+b}{c t+d}\right)\right) .
\end{aligned}
$$

Доказательство. Для функции $\psi(z, t)$ вида $(3.1)$ с $s=0$ имеем

$$
\begin{array}{r}
\Gamma(M) \psi(z, t)=\frac{1}{\sqrt{c t+d}} \exp \left(-\frac{1}{2}\left(\frac{1}{(c t+d)^{2}} h\left(\frac{a t+b}{c t+d}\right)+\frac{c}{(c t+d)}\right) z^{2}\right. \\
\left.+r\left(\frac{a t+b}{c t+d}\right)\right) \phi\left(\frac{z}{c t+d}, \frac{a t+b}{c t+d}\right)
\end{array}
$$

и $\phi\left(\frac{z}{c t+d}, \frac{a t+b}{c t+d}\right)=\frac{1}{(c t+d)^{\delta}} \hat{\phi}(z, t)$, где $\hat{\phi}(z, t)=z^{\delta}+\sum_{k \geqslant 2} \hat{\phi}_{k}(t) \frac{z^{2 k+\delta}}{(2 k+\delta) !}$ для $\hat{\phi}_{k}(t)=$ $\frac{1}{(c t+d)^{2 k}} \phi_{k}\left(\frac{a t+b}{c t+d}\right)$. Поскольку функция $\hat{\phi}(z, t)$ имеет вид $(3.2)$, действие на $h(t)$ и $e^{r(t)}$, индуцированное действием группы $\Gamma(M)$ на $\psi(z, t)$, имеет указанный в лемме вид.

\section{Общая конструкция.}

Теорема 3.2. Функиия $\psi(z, t)$ вида (3.1) является решением уравнения теплопроводности (2.1) тогда и только тогда, когда $s=0, r^{\prime}=-(\delta+1 / 2) h u$ функиия $\phi(z, t)$ является решением уравнения

$$
\frac{\partial}{\partial t} \phi=\mathscr{H}_{2} \phi-h \mathscr{H}_{0} \phi
$$

əəe

$$
\mathscr{H}_{2} \phi=\left(\frac{1}{2} \frac{\partial^{2}}{\partial z^{2}}+u z^{2}\right) \phi, \quad u=\frac{1}{2}\left(h^{\prime}+h^{2}\right), \quad \mathscr{H}_{0} \phi=\left(z \frac{\partial}{\partial z}-\delta\right) \phi
$$


Доказательство. Подставляя (3.1) и (3.2) в (2.1) и представляя правую и левую части уравнения в виде ряда по $z$, с точностью до элементов порядка $2 s+\delta$ получаем $(2 s+\delta)(2 s+\delta-1) e^{r(t)} z^{2 s+\delta-2}+\left(z^{2 s+\delta}\right)=0$; следовательно, $s=0$. С точностью до элементов порядка $\delta+2$ имеем $\left(\left(\delta+\frac{1}{2}\right) h(t)+r^{\prime}(t)\right) e^{r(t)} z^{\delta}+$ $\left(z^{\delta+2}\right)=0$; следовательно, $r^{\prime}(t)=-\left(\delta+\frac{1}{2}\right) h(t)$. Последнее утверждение теоремы получается подстановкой (3.1) в $(2.1)$ и делением на $e^{-\frac{1}{2} h(t) z^{2}+r(t)}$.

Положим $\boldsymbol{x}=\left(x_{2}, \ldots, x_{n+1}\right)$. Мы будем считать, что переменные $x_{q}$ имеют градуировку $-4 q$, а все постоянные (т. е. параметры, не зависящие от $t, z$ и $x$ ) имеют градуировку 0 , если не оговорено противное. Положим

$$
\Phi(z ; \boldsymbol{x})=z^{\delta}+\sum_{k \geqslant 2} \Phi_{k}(\boldsymbol{x}) \frac{z^{2 k+\delta}}{(2 k+\delta) !},
$$

где $z$ - переменная градуировки 2 , не зависящая от $\boldsymbol{x}$, и $\Phi_{k}(\boldsymbol{x})$ - однородные полиномы градуировки $-4 k$. Например, $\Phi_{2}(\boldsymbol{x})=c_{2} x_{2}$ и $\Phi_{3}(\boldsymbol{x})=c_{3} x_{3}$ для некоторых констант $c_{2}$ и $c_{3}$. Таким образом, $\Phi(z ; \boldsymbol{x})$ - однородная функция градуировки $2 \delta$. Заметим, что функции, однородные относительно некоторой градуировки, часто называют квазиоднородными. Мы не будем различать эти понятия, и все функции с такими свойствами будем называть однородными.

Из однородности функции $\Phi(z ; \boldsymbol{x})$ следует соотношение $\mathscr{H}_{0} \Phi=L_{0} \Phi$, где $L_{0}=$ $\sum_{k=2}^{n+1} 2 k x_{k} \frac{\partial}{\partial x_{k}}$.

Рассмотрим в $\mathbb{C}^{n}$ с координатами $\left(x_{2}, \ldots, x_{n+1}\right)$ набор однородных полиномов $p_{q}(\boldsymbol{x}) \mathrm{c} \operatorname{deg} p_{q}=-4 q, q=3, \ldots, n+2$, и введем однородную полиномиальную динамическую систему

$$
\frac{d x_{k}}{d \tau}=p_{k+1}(\boldsymbol{x}), \quad k=2, \ldots, n+1, \quad \operatorname{deg} \tau=4 .
$$

Положим $L_{2} \Phi=\sum p_{k+1}\left(x_{2}, \ldots, x_{n+1}\right) \frac{\partial}{\partial x_{k}} \Phi$. Нам потребуются следующие результаты:

Теорема 3.3. Функиия $\Phi(z ; \boldsymbol{x})$ удовлетворяет уравнению

$$
\mathscr{H}_{2} \Phi=L_{2} \Phi
$$

тогда и только тогда, когда

$$
\begin{gathered}
\Phi_{2}=-4(1+2 \delta) u, \quad \Phi_{3}=2 \frac{d}{d \tau} \Phi_{2}, \\
\Phi_{k+1}=2 \frac{d}{d \tau} \Phi_{k}+\frac{(2 k+\delta-1)(2 k+\delta)}{2(1+2 \delta)} \Phi_{2} \Phi_{k-1}, \quad k>2 .
\end{gathered}
$$

Теорема доказывается непосредственно подстановкой (3.4) в (3.6).

Следствие 3.4. В условиях теоремы для постоянной с, такой, что $\Phi_{2}(\boldsymbol{x})=$ $c x_{2}$, имеет место равенство $u=-\frac{c}{4(1+2 \delta)} x_{2}$.

Напомним, что функция $f(\boldsymbol{x})$ называется невырожденной, если для векторфункции $\phi(\boldsymbol{x})$ из равенства $\langle\phi(\boldsymbol{x}), \operatorname{grad} f(\boldsymbol{x})\rangle \equiv 0$ следует, что $\phi(\boldsymbol{x}) \equiv 0$.

Теорема 3.5. Пусть $\Phi(z ; \boldsymbol{x})$ - невырожденная функиия вида (3.4), коэффициенты которой удовлетворяют равенствам (3.7). Функиия

$$
\phi(z, t)=\Phi\left(z ; x_{2}(t), \ldots, x_{n+1}(t)\right)
$$


является решением уравнения (3.3) тогда и только тогда, когда вектор-функиия $\boldsymbol{x}(t)=\left(x_{2}(t), \ldots, x_{n+1}(t)\right)$ удовлетворяет динамической системе

$$
\frac{d}{d t} x_{k}(t)=p_{k+1}(\boldsymbol{x}(t))-2 k h(t) x_{k}(t), \quad k=2, \ldots, n+1 .
$$

Доказательство. После подстановки в него равенства (3.8) уравнение (3.3) принимает вид $\sum_{k=2}^{n+1}\left(\frac{\partial x_{k}}{\partial t}-p_{k+1}\left(x_{2}, \ldots, x_{n+1}\right)+2 k h x_{k}\right) \frac{\partial \Phi}{\partial x_{k}}=0$. Условие невырожденности функции $\Phi$ завершает доказательство.

Суммируем результаты этого раздела. Пусть $p_{k}(\boldsymbol{x}), k=3, \ldots, n+2,-$ однородные полиномы от $\boldsymbol{x}=\left(x_{2}, \ldots, x_{n+1}\right), \operatorname{deg} x_{q}=-4 q, \operatorname{deg} p_{k}=-4 k, \operatorname{deg} t=4$, $\operatorname{deg} h=-4, \operatorname{deg} r=0, c$ - константа, $\Phi(z ; \boldsymbol{x})$ - однородная функция вида $(3.4)$.

Теорема 3.6 (ключевая теорема). Из любых двух из трех следующих условий вытекает третъе:

1) функиия

$$
\psi(z, t)=e^{-\frac{1}{2} h(t) z^{2}+r(t)} \Phi(z ; \boldsymbol{x}(t))
$$

удовлетворяет уравнению теплопроводности (2.1);

2) коэфбициенты функции $\Phi(z ; \boldsymbol{x})$ задаются соотношением

$$
\Phi_{q}=2 \sum_{k=2}^{n+1} p_{k+1}(\boldsymbol{x}) \frac{\partial}{\partial x_{k}} \Phi_{q-1}+\frac{(2 q+\delta-3)(2 q+\delta-2)}{2(1+2 \delta)} \Phi_{2} \Phi_{q-2}, \quad q=4,5,6, \ldots,
$$

с начальными условиями $\Phi_{2}(\boldsymbol{x})=c x_{2}, \Phi_{3}=2 c p_{3}(\boldsymbol{x})$;

3) набор функиий $(r(t), h(t), \boldsymbol{x}(t))$ удовлетворяет однородной полиномиальной динамической системе

$$
\begin{gathered}
\frac{d}{d t} r=-\left(\delta+\frac{1}{2}\right) h, \quad \frac{d}{d t} h=-h^{2}-\frac{c}{2(1+2 \delta)} x_{2}, \\
\frac{d}{d t} x_{k}=p_{k+1}(\boldsymbol{x})-2 k h x_{k}, \quad k=2, \ldots, n+1 .
\end{gathered}
$$

Действие Г группы $S L(2, \mathbb{C})$ на решениях уравнения теплопроводности описано выше.

Следствие 3.7. Пусть $\psi(z, t)$ - решение уравнения теплопроводности вида (3.9). Тогда функиия

$$
\Gamma(M) \psi(z, t)=e^{-\frac{1}{2} \widehat{h}(t) z^{2}+\widehat{r}(t)} \Phi(z ; \widehat{\boldsymbol{x}}(t)),
$$

где $\widehat{h}(t)=\gamma_{1}(M) h(t), e^{\widehat{r}(t)}=\gamma_{2}(M) e^{r(t)} u \widehat{x}_{k}(t)=\frac{x_{k}(t)}{(c t+d)^{2 k}}$, является решением того же вида.

\section{§4. Редукция к обыкновенным дифференциальным уравнениям}

Для набора однородных полиномов $p_{k}(\boldsymbol{x})$ с $\operatorname{deg} p_{k}=-4 k, k=3, \ldots, n+2$, из градуировки следует, что для любого $k \leqslant n$ полином $p_{k}(\boldsymbol{x})$ не зависит от $x_{k+1}, \ldots, x_{n+1}$. Далее полиномы $p_{k}\left(x_{2}, \ldots, x_{n+1}\right)$, не зависящие от $x_{s}, \ldots, x_{n+1}$, мы также обозначаем через $p_{k}\left(x_{2}, \ldots, x_{s-1}\right)$.

Лемма 4.1. Группа полиномиалъных преобразований вида

$$
X_{2}=c_{2} x_{2}, \quad X_{k}=c_{k} x_{k}+q_{k}\left(x_{2}, \ldots, x_{k-1}\right), \quad k=3, \ldots, n+1,
$$


где $c_{k} \neq 0$ - постоянные $и q_{k}\left(x_{2}, \ldots, x_{k}\right)$ - однородные полиномы, $\operatorname{deg} q_{k}=$ $-4 k$, действует на пространстве однородных полиномиальных динамических систем вида (3.5). Это действие переводит систему (3.5) в систему

$$
\frac{d}{d \tau} X_{k}=P_{k+1}\left(X_{2}, \ldots, X_{n+1}\right), \quad k=3, \ldots, n+2,
$$

где

$$
P_{k+1}\left(X_{2}, \ldots, X_{n+1}\right)=c_{k} p_{k+1}\left(x_{2}, \ldots, x_{n+1}\right)-\frac{d}{d \tau} q_{k}\left(x_{2}, \ldots, x_{k-1}\right) .
$$

Замечание 4.2. Выражение $\frac{d}{d \tau} q_{k}\left(x_{2}, \ldots, x_{k-1}\right)$ является сокращением выражения $\sum_{q} \frac{\partial}{\partial x_{q}} q_{k}\left(x_{2}, \ldots, x_{k-1}\right) p_{q+1}\left(x_{2}, \ldots, x_{n+1}\right)$.

Лемма доказывается прямой подстановкой.

Лемма 4.3. Группа полиномиальных преобразований (4.1) действует на $\partial и$ намических системах вида (3.10) и переводит решение $(r(t), h(t), \boldsymbol{x}(t))$ такой системы в решение $\left(r(t), h(t), X_{2}(t), \ldots, X_{n+1}(t)\right)$ динамической системь

$$
\begin{gathered}
\frac{d}{d t} r=-\left(\delta+\frac{1}{2}\right) h, \quad \frac{d}{d t} h=-h^{2}-\frac{c}{2 c_{2}(1+2 \delta)} X_{2}, \\
\frac{d}{d t} X_{k}=P_{k+1}\left(X_{2}, \ldots, X_{n+1}\right)-2 k h X_{k}, \quad k=2, \ldots, n+1 .
\end{gathered}
$$

Доказательство. Каждое преобразование (4.1) можно представить как композицию преобразований вида

$$
X_{k}=c_{k} x_{k}, \quad c_{k} \neq 0, \quad k=2, \ldots, n+1,
$$

и

$$
X_{k}=x_{k}, \quad k \neq s, \quad X_{s}=x_{s}+a_{0} x_{2}^{j_{2}} \cdots x_{s-2}^{j_{s-2}}, \quad \text { где } \quad \sum_{m=2}^{s-2} m j_{m}=s, \quad a_{0}=\text { const } .
$$

При этом (4.3) переводит (3.10) в

$$
\begin{gathered}
\frac{d}{d t} r=-\left(\delta+\frac{1}{2}\right) h, \quad \frac{d}{d t} h=-h^{2}-\frac{c}{2(1+2 \delta) c_{2}} X_{2}, \\
\frac{d}{d t} X_{k}=c_{k} p_{k+1}\left(\frac{X_{2}}{c_{2}}, \ldots, \frac{X_{n+1}}{c_{n+1}}\right)-2 k h X_{k}, \quad k=2, \ldots, n+1,
\end{gathered}
$$

и (4.4) переводит (3.10) в

$$
\begin{gathered}
\frac{d}{d t} r=-\left(\delta+\frac{1}{2}\right) h, \quad \frac{d}{d t} h=-h^{2}-\frac{c}{2(1+2 \delta)} X_{2}, \\
\frac{d}{d t} X_{k}=P_{k+1}\left(X_{2}, \ldots, X_{n+1}\right)-2 k h X_{k}, \quad k=2, \ldots, n+1,
\end{gathered}
$$

где $P_{k+1}\left(X_{2}, \ldots, X_{n+1}\right)$ задается выражением (4.2). Второе утверждение леммы имеет место, так как из (3.10) следует, что функция $F=x_{2}^{j_{2}} \cdots x_{s-2}^{j_{s-2}}$, где $\sum_{m=2}^{s-2} m j_{m}=s$, удовлетворяет уравнению $\frac{d}{d t} F=\sum p_{k+1}(\boldsymbol{x}) \frac{\partial}{\partial x_{k}} F+2 s h F$. 
Лемма 4.4. Если $p_{s}(\boldsymbol{x})$ не зависит от $x_{s}$ для некоторого $s$, то набор $\left(r(t), h(t), x_{2}(t), \ldots, x_{s-1}(t)\right)$ является решением системы

$$
\begin{gathered}
\frac{d}{d t} r=-\left(\delta+\frac{1}{2}\right) h, \quad \frac{d}{d t} h=-h^{2}-\frac{c}{2(1+2 \delta)} x_{2}, \\
\frac{d}{d t} x_{k}=p_{k+1}\left(x_{2}, \ldots, x_{s-1}\right)-2 k h x_{k}, \quad k=2, \ldots, s-1 .
\end{gathered}
$$

Это решение вместе с набором полиномов $p_{k}\left(x_{2}, \ldots, x_{s-1}\right), k=3, \ldots, s$, задает решение уравнения теплопроводности, совпадающее с описанным выше решением (3.9).

Таким образом, в условиях леммы решение уравнения теплопроводности не зависит от полиномов $p_{s+1}, \ldots, p_{n+2}$.

Доказательство. Если $p_{s}\left(x_{2}, \ldots, x_{n+1}\right)$ не зависит от $x_{s}$, то все $p_{k}\left(x_{2}, \ldots\right.$, $\left.x_{n+1}\right), k=3, \ldots, s$, не зависят от $x_{s}, \ldots, x_{n+1}$ в силу градуировки и, следовательно, система (4.5) является подсистемой системы (3.10), зависящей только от переменных $h(t), r(t), x_{2}(t), \ldots, x_{s-1}(t)$. Функции $\Phi_{k}$ суть функции от $\left(h(t), r(t), x_{2}(t), \ldots, x_{s-1}(t)\right)$, так как $\Phi_{2}, p_{3}, \ldots, p_{s}$ суть такие функции, а $\Phi_{k}$ задаются рекуррентно формулой (3.7). Таким образом, решение (3.9) уравнения теплопроводности не зависит от $x_{s}(t), \ldots, x_{n+1}(t)$ и задается лишь решением системы (4.5).

Полиномиальная динамическая система (3.5) называется редуцированной, если она задается набором

$$
p_{k}(\boldsymbol{x})=x_{k}, \quad k=3, \ldots, n+1, \quad p_{n+2}(\boldsymbol{x})=P_{n}(\boldsymbol{x}),
$$

где $P_{n}(\boldsymbol{x})$ - однородный полином градуировки $-4(n+2)$.

Лемма 4.5. Каждое решение вида (3.9) уравнения теплопроводности можно получить, используя в нашем построении редуцированную динамическую систему (4.6) и решение системы

$$
\begin{aligned}
\frac{d}{d t} r & =-\left(\delta+\frac{1}{2}\right) h, \quad \frac{d}{d t} h=-h^{2}+x_{2}, \\
\frac{d}{d t} x_{k} & =x_{k+1}-2 k h x_{k}, \quad k=2, \ldots, n, \\
\frac{d}{d t} x_{n+1} & =P_{n}\left(x_{2}, \ldots, x_{n+1}\right)-2(n+1) h x_{n+1} .
\end{aligned}
$$

Доказательство. Начнем с набора однородных полиномов $p_{k}\left(x_{2}, \ldots, x_{n+1}\right)$, $k=3, \ldots, n+2$. Согласно лемме 4.4, можно предполагать, что $p_{k}\left(x_{2}, \ldots, x_{n+1}\right)$ существенно зависят от $x_{k}$ для $k=3, \ldots, n+1$; следовательно, в (4.1) можно положить $X_{2}=-\frac{c}{2(1+2 \delta)} x_{2}$ и $X_{k}=c_{k-1} p_{k}\left(x_{2}, \ldots, x_{n+1}\right)+\frac{d}{d \tau} q_{k-1}\left(x_{2}, \ldots, x_{k-1}\right)$ для $k=3, \ldots, n+1$, где $c_{k-1}$ и $q_{k-1}$ определены в (4.1). Такое преобразование приводит динамическую систему к требуемому виду.

Система (4.7) задается числом $n$ и полиномом $P_{n}(\boldsymbol{x})$. В этой системе $r$ определяется функцией $h(t)$ с точностью до постоянной интегрирования $r_{0}$ соотношением $r^{\prime}=-\left(\delta+\frac{1}{2}\right) h$, а $x_{2}, \ldots, x_{n+1}$ определены равенствами

$$
x_{2}=h^{\prime}+h^{2}, \quad x_{k}=x_{k-1}^{\prime}+2(k-1) h x_{k-1}, \quad k=3, \ldots, n+1 .
$$


Подставляя $x_{k}$ как функцию от $h(t)$ в $\frac{d}{d t} x_{n+1}=P_{n}\left(x_{2}, \ldots, x_{n+1}\right)-2(n+1) h x_{n+1}$, получаем обыкновенное дифференциальное уравнение

$$
\mathscr{D}_{P_{n}, n+1}(h)=0
$$

порядка $n+1$ на $h(t)$. Это уравнение однородно относительно градуировки $\operatorname{deg} h=-4, \operatorname{deg} t=4$.

Суммируем результаты этого параграфа.

Теорема 4.6. Каждое решение вида (3.9) уравнения теплопроводности задается набором $\left(n, P_{n}, h, r_{0}\right)$, где $n-$ натуральное число, $P_{n}$ - однородный полином $P_{n}(\boldsymbol{x})$ градуировки $-4(n+2), h-$ решение $h(t)$ уравнения $\mathscr{D}_{P_{n}, n+1}(h)=$ $0, r_{0}-$ константа.

Следствие 4.7. Каждое решение вида (3.9) уравнения теплопроводности задается конечномерным числовым вектором $\left(n, \boldsymbol{P}_{n}, \boldsymbol{h}_{n}, r_{0}\right)$, где $\boldsymbol{P}_{n}-$ вектор коэфбициентов полинома $P_{n}(\boldsymbol{x})$ и $\boldsymbol{h}_{n}$ - вектор начальных данных задачи Коши уравнения (4.9).

\section{§5. Обыкновенные дифференциальные уравнения, ассоциированные с уравнением теплопроводности}

Путь $P_{n}(\boldsymbol{y})$ - полином $n$-мерного аргумента, такой, что для градуированного вектора $\boldsymbol{x}=\left(x_{2}, \ldots, x_{n+1}\right)$ полином $P_{n}(\boldsymbol{x})$ является однородным градуировки $-4(n+2)$.

Обозначим через $V_{n}$ линейное векторное пространство таких полиномов.

Лемма 5.1. Имеем $\operatorname{dim} V_{n}=p(n+2)-p(n+1)-1$, где $p(n)-$ число разбиений числа $n$.

Доказательство. Размерность пространства однородных полиномов градуировки $-4(n+1)$ от переменных $x_{1}, \ldots, x_{n+1}$ с $\operatorname{deg} x_{k}=-4 k$ равна $p(n+1)$. Следовательно, $p(n+2)-p(n+1)$ - размерность пространства полиномов от $x_{1}, \ldots, x_{n+2}$ градуировки $-4(n+2)$, которые нельзя представить в виде $x_{1} P$, где $P$ - полином градуировки $-4(n+1)$. Учитывая полиномы $p\left(x_{1}, \ldots, x_{n+2}\right)=$ $c x_{n+2}$, получаем требуемую размерность.

Рассмотрим уравнение (4.9). В случае $P_{n} \equiv 0$ положим $\mathscr{D}_{0, n+1}(h)=\mathscr{D}_{n+1}(h)$.

Теорема 5.2. Имеем $\mathscr{D}_{1}(h)=\left(\frac{d}{d t}+h\right) h u \mathscr{D}_{n}(h)=\left(\frac{d}{d t}+2 n h\right) \mathscr{D}_{n-1}(h) \partial_{\Omega я}$ каждого $n>1$.

Доказательство. Уравнение $\mathscr{D}_{n+1}=0$ получается из $\frac{d x_{n+1}}{d t}=-2(n+1) h x_{n+1}$ подстановкой (4.8). Для $n=0$ подстановку делать не нужно и уравнение имеет вид $h^{\prime}+h^{2}=0$. Переход от $\mathscr{D}_{n+1}$ к $\mathscr{D}_{n+2}$ задается подстановкой $x_{n+2}=x_{n+1}^{\prime}+$ $2(n+1) h x_{n+1}$ в $\frac{d x_{n+2}}{d t}=-2(n+2) h x_{n+2}$, т. е. действием оператора $\frac{d}{d t}+2(n+2) h$ на $\mathscr{D}_{n+1}(h)$.

Следствие 5.3. Для $n>1$ имеем $\mathscr{D}_{n}(h)=h^{(n)}+n(n+1) h^{(n-1)} h+\cdots$ $+2^{n-1} n ! h^{n+1}$.

Теорема 5.4. Для $(n-1)$-мерной вектор-функиии $\mathscr{D}(h)=\left(\mathscr{D}_{1}(h), \ldots\right.$, $\left.\mathscr{D}_{n-1}(h)\right)$ и полинома $P_{n}(\boldsymbol{y}) \in V_{n}$ имеем $\mathscr{D}_{P_{n}, n+1}(h)=\mathscr{D}_{n+1}(h)-P_{n}(\mathscr{D}(h))$.

Доказательство следует из (4.7) и использует теорему 5.2.

Положим $d=\sum_{k=1}^{\infty} y_{k+1} \frac{\partial}{\partial y_{k}}$. 
Теорема 5.5. Для полинома $P_{n}(\boldsymbol{x}) \in V_{n}$ имеем $\left(\frac{d}{d t}+2(n+2) h\right) \mathscr{D}_{P_{n}, n+1}(h)$ $=\mathscr{D}_{P_{n+1}, n+2}(h)$, где $P_{n+1}(\boldsymbol{y})=d P_{n}(\boldsymbol{y})$.

Доказательство. Для каждого монома $\mathscr{D}_{1}(h)^{j_{1}} \ldots \mathscr{D}_{n}(h)^{j_{n}}$ в $\left.P_{n}(\mathscr{D}(h))\right)$

$$
\begin{aligned}
\left(\frac{d}{d t}\right. & +2(n+2) h) \mathscr{D}_{1}(h)^{j_{1}} \ldots \mathscr{D}_{n}(h)^{j_{n}} \\
& =\sum_{k} j_{k} \mathscr{D}_{1}(h)^{j_{1}} \ldots \mathscr{D}_{k}(h)^{j_{k}-1} \ldots \mathscr{D}_{n}(h)^{j_{n}}\left(\frac{d}{d t}+2(k+1) h\right) \mathscr{D}_{k}(h) \\
& =\sum_{k} j_{k} \mathscr{D}_{1}(h)^{j_{1}} \ldots \mathscr{D}_{k}(h)^{j_{k}-1} \ldots \mathscr{D}_{n}(h)^{j_{n}} \mathscr{D}_{k+1}(h) .
\end{aligned}
$$

Следовательно, $\left.\left(\frac{d}{d t}+2(n+2) h\right) P_{n}(\mathscr{D}(h))\right)=\left(d P_{n}\right)(\mathscr{D}(h))$.

Для $n>1$ мы получаем семейства уравнений, параметрами которых являются коэффициенты полиномов $P_{n}$. Для фиксированных значений этих параметров мы получаем специальные классы дифференциальных уравнений. Далее мы рассмотрим в деталях уравнения $\mathscr{D}_{P_{n}, n+1}(h)=0$, где $n=0,1,2,3,4$, и обсудим получающиеся классы уравнений.

Говорят, что обыкновенное дифференциальное уравнение обладает свойством Пенлеве, если можно построить однозначное общее решение этого уравнения. Характеристикой уравнения со свойством Пенлеве является независимость его критических особых точек от начальных данных (см. [3]). Уравнения со свойством Пенлеве играют важную роль в современных физических задачах. Задачи, связанные с уравнением Шази-3, описаны в [4]-[6]. Действие группы $S L(2, \mathbb{C})$ на пространстве его решений изучено в [7]. Далее мы покажем что для некоторых параметров уравнение $\mathscr{D}_{P_{2}, 3}(h)=0$ совпадает с уравнениями Шази-3 и Шази-12 и, следовательно, обладает свойством Пенлеве.

Согласно следствию 3.7 , группа $S L(2, \mathbb{C})$ действует на пространстве решений каждого дифференциального уравнения $\mathscr{D}_{P_{n}, n+1}(h)=0$.

\section{§6. Дискретные дифференциальные уравнения, ассоциированные с уравнением теплопроводности}

В нашей общей конструкции (§3) мы построили однородные функции $\Phi(z ; \boldsymbol{x})$, удовлетворяющие уравнению (3.6) и, следовательно, являющиеся решением уравнения теплопроводности (см. теорему 3.6). В этом параграфе мы вернемся к проблеме построения таких функций.

Однородная функция $\Phi(z ; \boldsymbol{x})$ (см. (3.4)) может быть представлена в виде

$$
\Phi(z ; \boldsymbol{x})=z^{\delta}+\sum_{\|J\| \geqslant 4} a(J) \boldsymbol{x}^{J} \frac{z^{\|J\|+\delta}}{(\|J\|+\delta) !},
$$

где, как и раньше, $\boldsymbol{x}=\left(x_{2}, \ldots, x_{n+1}\right), \operatorname{deg} x_{k}=-4 k, \operatorname{deg} z=2, J=\left(j_{2}, \ldots, j_{n+1}\right)$ - мультииндекс, $a(J)$ - постоянные коэффициенты и $\|J\|=\sum_{k=2}^{n+1} 2 k j_{k}$. То есть в обозначениях, приведенных выше, $\Phi_{k}(x)=\sum_{\|J\|=2 k} a(J) \boldsymbol{x}^{J}$.

Для редуцированной динамической системы (4.6) уравнение (3.6) имеет вид

$$
\left(\frac{1}{2} \frac{\partial^{2}}{\partial z^{2}}+u z^{2}\right) \Phi=P_{n}(\boldsymbol{x}) \frac{\partial}{\partial x_{n+1}} \Phi+\sum_{k=2}^{n} x_{k+1} \frac{\partial}{\partial x_{k}} \Phi,
$$


где $u=-\frac{c}{4(1+2 \delta)} x_{2}$ и $c$ - такая константа, что $\Phi_{2}=c x_{2}$. Заметим, что в предыдущих параграфах мы использовали значение $c=-2(1+2 \delta)$. Представим однородные полиномы $P_{n}(\boldsymbol{x})$ в виде $P_{n}(\boldsymbol{x})=\sum_{S} p(S) \boldsymbol{x}^{S}$ с мультииндексом $S$. Имеем $\|S\|=2(n+2)$.

Пример 6.1. Для $n=1$ имеем $J=\left(j_{2}\right)$. Коэффициенты $a\left(j_{2}\right)$ определяются рекуррентной формулой

$$
a\left(j_{2}\right)=\frac{c}{2(1+2 \delta)}\left(4 j_{2}+\delta-3\right)\left(4 j_{2}+\delta-2\right) a\left(j_{2}-1\right)
$$

с начальным условием $a(0)=1$.

Пример 6.2. Для $n=2$ имеем $J=\left(j_{2}, j_{3}\right)$. Коэффициенты $a\left(j_{2}, j_{3}\right)$ определяются рекуррентной формулой

$$
\begin{aligned}
a(J)=\frac{c}{2(1+2 \delta)}(\|J\| & +\delta-3)(\|J\|+\delta-2) a(J-(1,0)) \\
& +2\left(j_{2}+1\right) a(J+(1,-1))+2\left(j_{3}+1\right) p(2,0) a(J+(-2,1))
\end{aligned}
$$

с начальными условиями $a(0,0)=1$ и $a\left(j_{2}, j_{3}\right)=0$ при $j_{2}<0$ и при $j_{3}<0$.

Положим $T_{1} a\left(j_{2}, j_{3}\right)=a\left(j_{2}-1, j_{3}\right), T_{2} a\left(j_{2}, j_{3}\right)=a\left(j_{2}+1, j_{3}-1\right)$. Тогда эта рекуррентная формула эквивалентна дискретному дифференциальному уравнению $W a\left(j_{2}, j_{3}\right)=0$ с оператором

$W=\frac{c}{2(1+2 \delta)}(\|J\|+\delta-3)(\|J\|+\delta-2) T_{1}+2\left(j_{2}+1\right) T_{2}+2\left(j_{3}+1\right) p(2,0) T_{1} T_{2}^{-1}-1$

и начальными условиями $a(0,0)=1, a\left(j_{2}, j_{3}\right)=0$ при $\min \left(j_{2}, j_{3}\right)<0$.

Положим $T_{1} a(J)=a\left(j_{2}-1, j_{3}, \ldots, j_{n+1}\right), T_{k} a(J)=a\left(j_{2}, \ldots, j_{k}+1, j_{k+1}-1\right.$, $\left.\ldots, j_{n+1}\right)$, где $k=2, \ldots, n$, и

$T_{S} a(J)=T_{1}^{s_{2}+s_{3}+\cdots+s_{n+1}-1} T_{2}^{s_{3}+\cdots+s_{n+1}-1} \cdots T_{n}^{s_{n+1}-1} a(J)=T_{1}^{-1} \cdots T_{n}^{-1} a(J-S)$.

Теорема 6.3. Коэффициенты $а(J)$ являются решениями дискретного дифференциального уравнения $W a(J)=0$ с оператором

$W=\frac{c}{2(1+2 \delta)}(\|J\|+\delta-2)(\|J\|+\delta-3) T_{1}+\sum_{k=2}^{n} 2\left(j_{k}+1\right) T_{k}+2 \sum_{S}\left(j_{n+1}+1\right) p(S) T_{S}-1$

и начальными условиями $а(0,0)=1$ и а $(J)=0$ при $\min \left(j_{k}\right)<0$. Из уравнения $W a(J)=0$ коэфбициенты $а(J)$ выражсатся как линейные комбинации коэфбициентов а $\left(J^{\prime}\right)$, таких, что $\left\|J^{\prime}\right\|<\|J\|$.

Доказательство осуществляется прямой подстановкой (6.1) в (6.2), причем используется, что $\|S\|=2(n+2)$.

Соответствующее дискретное дифференциальное уравнение для ряда сигмафункции Вейерштрасса получено в [11].

Следствие 6.4. Для $c \geqslant 0 u p(S) \geqslant 0$ для всех $S$ получаем $a(J) \geqslant 0$ для любого $\mathrm{J}$.

Рядом Гурвица над коммутативным ассоциативным кольцом $A$ называется формальный степенной ряд вида

$$
\varphi(z)=\sum_{k \geqslant 0} \varphi_{k} \frac{z^{k}}{k !} \in A \otimes \mathbb{Q}[[z]], \quad \text { где } \varphi_{k} \in A .
$$


Следствие 6.5. Если $\frac{c}{(1+2 \delta)} \in \mathbb{Z} u p(S) \in \mathbb{Z}$, то $\Phi(z ; \boldsymbol{x})$ является рядом Гурвииа по в над кольи,ом $\mathbb{Z}[\boldsymbol{x}]$.

\section{§7. Рациональные решения}

В этом параграфе мы описываем дифференциальные уравнения, приводящие к рациональным решениям уравнений вида $\mathscr{D}_{P_{n}, n+1}=0$.

Для функции $h=h(t)$ и постоянной $b$ рассмотрим $(n+2) \times(n+2)$-матрицу

$$
S_{n}(h)=\left(\begin{array}{cccc}
b h & -1 & \ldots & 0 \\
b h^{\prime} & b h & \ldots & 0 \\
\vdots & \vdots & & \\
\frac{b}{n !} h^{(n)} & \frac{b}{(n-1) !} h^{(n-1)} & \ldots & -(n+1) \\
\frac{b}{(n+1) !} h^{(n+1)} & \frac{b}{n !} h^{(n)} & \ldots & b h
\end{array}\right) .
$$

Уравнение

$$
\frac{1}{b} \operatorname{det} S_{n}(h)=0
$$

является обыкновенным дифференциальным уравнением, однородным относительно градуировки $\operatorname{deg} h=-4, \operatorname{deg} t=4$. Имеем $\frac{1}{b} \operatorname{det} S_{0}(h)=h^{\prime}+b h^{2}$ и $\frac{1}{b} \operatorname{det} S_{n}(h)=h^{(n+1)}+(n+2) b h h^{(n)}+\cdots+b^{n+1} h^{n+2}$ для $n>0$.

Теорема 7.1. Для любого челочисленного $n \geqslant 0$ функция

$$
h(t)=h_{n}(t)=\frac{1}{b} \sum_{k=1}^{n+1} \frac{1}{t-a_{k}}
$$

является общим решением уравнения (7.1).

Доказательство. Рассмотрим полиномы Ньютона $N_{q}=\sum_{k=1}^{n+1} z_{k}^{q}$ от $n+1$ переменных, где $z_{k}(t)=\frac{1}{a_{k}-t}$. Имеем $(-1)^{q} \frac{b}{q !} h^{(q)}(t)=N_{q+1}$. Используя классический результат теории симметрических функций, получаем $\operatorname{det} S_{n}(h)=$ $(n+2) ! s_{n+2}\left(z_{1}, \ldots, z_{n+1}, 0\right) \equiv 0$, где $s_{n+2}(\cdot)$ - элементарная симметрическая функция. Следовательно, функция (7.2) является решением дифференциального уравнения (7.1) порядка $n+1$. Она определяет общее решение этого уравнения, поскольку зависит от $n+1$ параметров $a_{1}, \ldots, a_{n+1}$.

Следствие 7.2. Для каждого положительного целого $n$ для существования полинома $P_{n}$, такого, что $\mathscr{D}_{P_{n}, n+1}(h)=\frac{1}{b} \operatorname{det} S_{n}(h)$, необходимо выполнение условия $b=n+1$.

Вычисления для $n=0,1,2,3,4$ показывают, что это условие также является достаточным (см. ниже).

Задача 7.3. Доказать, что в условиях следствия условие $b=n+1$ является достаточным.

\section{§8. Частные случаи}

В этом параграфе мы детально обсуждаем для случаев $n=0,1,2,3,4$ следствия полученных выше результатов. 
8.1. Случай $\boldsymbol{n}=\mathbf{0}$. Имеем $\mathscr{D}_{1}(h)=h^{\prime}+h^{2}$. В этом случае нет переменных $x_{k}$ и $P_{0}=0$. Система (4.7) принимает вид

$$
\frac{d}{d t} r=-\left(\delta+\frac{1}{2}\right) h, \quad \frac{d}{d t} h=-h^{2} .
$$

Общее решение этой системы: $h(t)=\frac{a}{a t-b}, r(t)=-\left(\frac{1}{2}+\delta\right) \ln (a t-b)+r_{0}$, где параметры $r_{0} \in \mathbb{C}$ и $(a: b) \in \mathbb{C} P^{1}-$ константы. Следовательно, функция

$$
\psi(z, t)=\frac{\exp r_{0}}{(a t-b)^{1 / 2+\delta}} \exp \left(-\frac{a z^{2}}{2(a t-b)}\right) z^{\delta}
$$

является решением уравнения теплопроводности. В случае $\delta=0, r_{0}=0, b / a=c$ она совпадает с решением (2.4) уравнения теплопроводности.

Следствие 8.1. Для $\delta=0 u b / a<t<\infty$ функиия $\psi(z, t)$ является гауссовой функиией плотности распределения.

8.2. Случай $\boldsymbol{n}=\mathbf{1}$. Имеем $\mathscr{D}_{2}(h)=\left(\frac{d}{d t}+4 h\right) \mathscr{D}_{1}(h)$ и $\operatorname{deg} x_{2}=-8, \operatorname{deg} P_{1}=$ -12 ; следовательно, $P_{1}\left(x_{2}\right) \equiv 0$. Система (4.7) имеет вид

$$
\frac{d}{d t} r=-\left(\delta+\frac{1}{2}\right) h, \quad \frac{d}{d t} h=-h^{2}+x_{2}, \quad \frac{d}{d t} x_{2}=-4 h x_{2} .
$$

Таким образом, $h(t)$ является решением уравнения

$$
h^{\prime \prime}+6 h h^{\prime}+4 h^{3}=0 .
$$

Замечание 8.2. Дифференцируя (8.1) и подставляя $y(t)=2 h(t)$, получаем уравнение Шази-4:

$$
y^{\prime \prime \prime}=-3 y y^{\prime \prime}-3 y^{\prime 2}-3 y^{2} y^{\prime} .
$$

Общее решение уравнения (8.1) имеет вид $h(t)=\frac{1}{2}\left(\frac{1}{t-a}+\frac{1}{t-b}\right)$, и, следовательно, $x_{2}(t)=-\frac{1}{4}\left(\frac{1}{t-a}-\frac{1}{t-b}\right)^{2}$. В случае $a=b$ приходим к случаю $n=0$.

Согласно ключевой теореме, $\Phi\left(z ; x_{2}\right)=z^{\delta}+\sum_{q=1}^{\infty} \Phi_{2 q}\left(x_{2}\right) \frac{z^{4 q+\delta}}{(4 q+\delta) !}$, где $\Phi_{2}=$ $-2(1+2 \delta) x_{2}, \Phi_{2 q}=-(4 q+\delta-3)(4 q+\delta-2) x_{2} \Phi_{2 q-2}, q=2,3, \ldots$

Заметим, что функция $\Phi\left(z ; x_{2}\right)$ является решением уравнения $\frac{d^{2}}{d z^{2}} \Phi\left(z ; x_{2}\right)=$ $-x_{2} z^{2} \Phi\left(z ; x_{2}\right)$.

Лемма 8.3. $\Phi\left(z ; x_{2}\right)=z^{\delta} \gamma\left(z^{4} ; x_{2}, \delta\right)$, где $\gamma\left(v ; x_{2}, \delta\right)$ - решение дифберенииального уравнения

$$
\gamma^{\prime}(v)+\frac{4 v}{3+2 \delta} \gamma^{\prime \prime}(v)=\lambda \gamma(v), \quad \lambda=-\frac{1}{4(3+2 \delta)} x_{2},
$$

с начальным условием $\gamma(0)=1$.

Следовательно, функция $\gamma(v)$ является собственной функцией обобщенного оператора сдвига, определенного генератором

$$
d=\frac{d}{d v}+\frac{4}{3+2 \delta} v \frac{d^{2}}{d v^{2}} .
$$

8.3. Случай $\boldsymbol{n}=\mathbf{2}$. Этот случай приводит к замечательным уравнениям, обладающим свойством Пенлеве (см., например, [3]). 
Имеем $P_{2}\left(x_{2}, x_{3}\right)=c_{4} x_{2}^{2}$, где $c_{4}-$ константа, и $\mathscr{D}_{3}(h)=\left(\frac{d}{d t}+6 h\right) \mathscr{D}_{2}(h)$, $\mathscr{D}_{P_{2}, 3}(h)=\mathscr{D}_{3}(h)-c_{4} \mathscr{D}_{1}(h)^{2}$.

Система (4.7) имеет вид

$\frac{d}{d t} r=-\left(\delta+\frac{1}{2}\right) h, \quad \frac{d}{d t} h=-h^{2}+x_{2}, \quad \frac{d}{d t} x_{2}=x_{3}-4 h x_{2}, \quad \frac{d}{d t} x_{3}=c_{4} x_{2}^{2}-6 h x_{3}$.

Следовательно, функция $h(t)$ является решением уравнения

$$
h^{\prime \prime \prime}+12 h h^{\prime \prime}-18\left(h^{\prime}\right)^{2}+\left(24-c_{4}\right)\left(h^{\prime}+h^{2}\right)^{2}=0 .
$$

Уравнение (8.2) переводится подстановкой $y(t)=-6 h(t)$ в

$$
y^{\prime \prime \prime}=2 y y^{\prime \prime}-3\left(y^{\prime}\right)^{2}+\frac{24-c_{4}}{216}\left(6 y^{\prime}-y^{2}\right)^{2} .
$$

Для $\frac{24-c_{4}}{216}=-\frac{4}{k^{2}-36}$ уравнение (8.3) является уравнением Шази-12. Для $c_{4}=24$ это уравнение является уравнением Шази- 3 .

Для $c_{4}=6$ уравнение (8.3) принимает вид

$$
y^{\prime \prime \prime}=2 y y^{\prime \prime}-y^{2} y^{\prime}+\frac{1}{12} y^{4} .
$$

Уравнение (8.4) является линейным относительно производных.

Имеем $\frac{1}{b} \operatorname{det} S_{2}(h)=h^{\prime \prime \prime}+4 b h h^{\prime \prime}+3 b\left(h^{\prime}\right)^{2}+6 b^{2} h^{2} h^{\prime}+b^{3} h^{4}$. Для $b=3$ получаем уравнение (8.2) с $c_{4}=-3$.

Следствие 8.4. Функция $-2\left(\frac{1}{t-a_{1}}+\frac{1}{t-a_{2}}+\frac{1}{t-a_{3}}\right)$ является общим решением уравнения Шази-12 (см. (8.3)) с $c_{4}=-3\left(k^{2}=4\right)$.

Мы опишем решения уравнения теплопроводности в случае, когда $y(t)=$ $-6 h(t)$ - решение уравнения Шази-3. Такие решения могут быть явно построены в терминах сигма-функции Вейерштрасса.

Определение 8.5. $\sigma$-функиией Вейерштрасса называется целая нечетная функция $\sigma(z)=\sigma\left(z ; g_{2}, g_{3}\right)$, такая, что $\left(\ln \sigma\left(z ; g_{2}, g_{3}\right)\right)^{\prime \prime}=-\wp\left(z ; g_{2}, g_{3}\right)$ и $\lim _{z \rightarrow 0}\left(\frac{\sigma(z)}{z}\right)=1$.

Здесь $\wp\left(z ; g_{2}, g_{3}\right)$ есть $\wp$-функция Вейерштрасса. Необходимые факты из теории эллиптических функций см., например, в [8].

Теорема $8.6[1]$. Функиия $\psi(z, t)=e^{-\frac{1}{2} h(t) z^{2}+r(t)} \sigma\left(z ; g_{2}(t), g_{3}(t)\right)$ удовлетворяет уравнению теплопроводности (2.1) тогда и только тогда, когда функиии $r(t), h(t), g_{2}(t)$ и $g_{3}(t)$ удовлетворяют однородной полиномиальной динамической системе в $\mathbb{C}^{4}$ с координатами $\left(r, h, g_{2}, g_{3}\right), \operatorname{deg} h=-4, \operatorname{deg} r=0$ :

$$
r^{\prime}=-\frac{3}{2} h, \quad h^{\prime}=-h^{2}+\frac{1}{12} g_{2}, \quad g_{2}^{\prime}=6 g_{3}-4 h g_{2}, \quad g_{3}^{\prime}=\frac{1}{3} g_{2}^{2}-6 h g_{3} .
$$

Пример 8.7. Функция

$$
\psi(z, t)=\exp \left(-\frac{1}{2} a^{2} t\right) \frac{\sin a z}{a}, \quad a=\text { const },
$$

является периодической нечетной функцией от $z$ с начальными условиями $\psi(0, t)=0, \psi^{\prime}(0, t)=\exp \left(-\frac{1}{2} a^{2} t\right)$. Она является классическим решением уравнения теплопроводности. В этом случае $r=-\frac{1}{2} a^{2} t, h=\frac{1}{3} a^{2}, g_{2}=\frac{4}{3} \gamma^{4}$, $g_{3}=\frac{8}{27} \gamma^{6}$. 
Пример 8.8. Для классического решения

$$
\psi(z, t)=\psi_{*}(z, t)-\psi_{*}(-z, t), \quad \text { где } \psi_{*}(z, t)=\frac{1}{\sqrt{t}} \exp \left(-\frac{(z-a)^{2}}{2 t}\right),
$$

убывающего при $z \rightarrow \pm \infty$, имеем $\gamma=-\frac{i a}{t}$ и $h=-\frac{a^{2}-3 t}{3 t^{2}}, r=\ln \left(\frac{2 a}{\sqrt{t^{3}}}\right)-\frac{a^{2}}{2 t}$, $g_{2}=\frac{4}{3} \frac{a^{4}}{t^{4}}, g_{3}=-\frac{8}{27} \frac{a^{6}}{t^{6}}$. Для этого решения $\psi(0, t)=0$ и $\psi^{\prime}(z, t)=\frac{2 a}{t \sqrt{t}} \exp \left(-\frac{a^{2}}{2 t}\right)$.

Рассмотрим динамическую систему (8.5). Она соответствует системе

$$
\frac{d}{d \tau} g_{2}=6 g_{3}, \quad \frac{d}{d \tau} g_{3}=\frac{1}{3} g_{2}^{2} .
$$

Для $x_{2}=\frac{1}{12} g_{2}, x_{3}=\frac{1}{2} g_{3}$ получаем случай $n=2, \delta=1, c_{4}=24$.

В этом случае уравнение (8.2) принимает вид $h^{\prime \prime \prime}+12 h h^{\prime \prime}-18\left(h^{\prime}\right)^{2}=0$ и приводится подстановкой $y(t)=-6 h(t)$ к уравнению Шази-3: $y^{\prime \prime \prime}=2 y y^{\prime \prime}-3\left(y^{\prime}\right)^{2}$.

Система (8.6) обладает решением

$$
g_{2}(\tau)=3 \wp\left(\tau+d ; 0, b_{3}\right), \quad g_{3}(\tau)=\frac{1}{2} \wp^{\prime}\left(\tau+d ; 0, b_{3}\right),
$$

где $b_{3}=\frac{4}{27} g_{2}(0)^{3}-4 g_{3}(0)^{2}$ и $d$ является решением совместной системы $\wp\left(d ; 0, b_{3}\right)$ $=\frac{1}{3} g_{2}(0), \wp^{\prime}\left(d ; 0, b_{3}\right)=2 g_{3}(0)$.

В терминах нашей конструкции $x_{2}=\frac{1}{4} \wp\left(\tau+d ; 0, b_{3}\right)$ для $n=2, c_{4}=24$. Далее мы покажем, что для $n=3$ возникают решения уравнения теплопроводности с $x_{2}=\frac{1}{4} \wp\left(\tau+d ; b_{2}, b_{3}\right)$.

Соответствующую функцию $\Phi(z ; \boldsymbol{x})$ можно разложить в ряд по параметру $b_{2}$. При $b_{2}=0$ получаем сигма-функцию Вейерштрасса (см. случай $n=2$ ).

8.4. Случай $\boldsymbol{n}=3$. В этом случае $\mathscr{D}_{4}(h)=\left(\frac{d}{d t}+8 h\right) \mathscr{D}_{3}(h)$ и $P_{3}\left(x_{2}, x_{3}, x_{4}\right)=$ $c_{5} x_{2} x_{3}$, где $c_{5}$ - константа и $\mathscr{D}_{P_{3}, 4}(h)=\mathscr{D}_{4}(h)-c_{5} \mathscr{D}_{1}(h) \mathscr{D}_{2}(h)$.

Система (4.7) принимает вид

$$
\begin{gathered}
\frac{d}{d t} r=-\left(\delta+\frac{1}{2}\right) h, \quad \frac{d}{d t} h=-h^{2}+x_{2}, \quad \frac{d}{d t} x_{2}=x_{3}-4 h x_{2}, \\
\frac{d}{d t} x_{3}=x_{4}-6 h x_{3}, \quad \frac{d}{d t} x_{4}=c_{5} x_{2} x_{3}-8 h x_{4} .
\end{gathered}
$$

Следовательно, $h(t)$ является решением уравнения $h^{\prime \prime \prime \prime}+20 h h^{\prime \prime \prime}-24 h^{\prime} h^{\prime \prime}+96 h^{2} h^{\prime \prime}-144 h\left(h^{\prime}\right)^{2}+\left(48-c_{5}\right)\left(h^{\prime}+h^{2}\right)\left(h^{\prime \prime}+6 h h^{\prime}+4 h^{3}\right)=0$.

Лемма 8.9. Для $c_{5}=2 c_{4}$ имеем $\mathscr{D}_{P_{3}, 4}(h)=\left(\frac{d}{d t}+8 h\right) \mathscr{D}_{P_{2}, 3}(h)$.

Следовательно, для $c_{5}=48$ уравнение (8.8) получается из уравнения Шази-3 подстановкой $y(t)=-6 h(t)$ и действием оператора $\frac{d}{d t}+8 h$.

Лемма 8.10. Для $c_{5}=24 u y(t)=-2 h(t)$ уравнение (8.8) принимает вид

$$
y^{\prime \prime \prime \prime}-10 y y^{\prime \prime \prime}+30 y^{2} y^{\prime \prime}-30 y^{3} y^{\prime}+6 y^{5}=0 .
$$

Уравнение (8.9) является линейным относительно производных.

Имеем $\frac{1}{b} \operatorname{det} S_{3}(h)=h^{\prime \prime \prime \prime}+5 b h h^{\prime \prime \prime}+10 b h^{\prime} h^{\prime \prime}+10 b^{2} h^{2} h^{\prime \prime}+15 b^{2} h\left(h^{\prime}\right)^{2}+10 b^{3} h^{3} h^{\prime}+$ $b^{4} h^{5}$. Для $b=4$ получаем уравнение (8.8) с $c_{5}=-16$. 
Следствие 8.11. Функиия

$$
\frac{1}{4}\left(\frac{1}{t-a_{1}}+\frac{1}{t-a_{2}}+\frac{1}{t-a_{3}}+\frac{1}{t-a_{4}}\right)
$$

является общим решением уравнения (8.8) с $c_{5}=-16$.

Рассмотрим функции (8.7) (случай $n=2$ ) как предел функций

$$
g_{2}(\tau)=3 \wp\left(\tau+d ; b_{2}, b_{3}\right), \quad g_{3}(\tau)=\frac{1}{2} \wp^{\prime}\left(\tau+d ; b_{2}, b_{3}\right)
$$

при $b_{2} \rightarrow 0$. Функции (8.10) вместе с $g_{4}=-b_{2} / 8$ задают общее решение системы

$$
\frac{d}{d \tau} g_{2}=6 g_{3}, \quad \frac{d}{d \tau} g_{3}=\frac{1}{3} g_{2}^{2}+2 g_{4}, \quad \frac{d}{d \tau} g_{4}=0 .
$$

Для $x_{2}=\frac{1}{12} g_{2}, x_{3}=\frac{1}{2} g_{3}, x_{4}=\frac{1}{6} g_{2}^{2}+g_{4}$ эта система

$$
\frac{d}{d \tau} x_{2}=x_{3}, \quad \frac{d}{d \tau} x_{3}=x_{4}, \quad \frac{d}{d \tau} x_{4}=-96 x_{2} x_{3},
$$

соответствует случаю $n=3, c_{5}=48$.

Следовательно, решение $\psi(z, t)$ уравнения теплопроводности, соответствующее этому случаю, имеет параметр $b_{2}$ и при $b_{2}=0$ является решением, соответствующим случаю $n=2, c_{4}=24$.

Таким образом, функция $e^{\frac{1}{2} h(t) z^{2}-r(t)} \psi(z, t)$ при $t=0$ является деформацией сигма-функции Вейерштрасса $\sigma\left(z ; g_{2}, g_{3}\right)$ с параметром деформации $b_{2}$.

8.5. Случай $\boldsymbol{n}=4$. В этом случае $\mathscr{D}_{5}(h)=\left(\frac{d}{d t}+10 h\right) \mathscr{D}_{4}(h)$ и $P_{4}\left(x_{2}, x_{3}, x_{4}, x_{5}\right)$ $=c_{6,2} x_{2}^{3}+c_{6,3} x_{3}^{2}+c_{6,4} x_{2} x_{4}$, где $c_{6,2}, c_{6,3}$ и $c_{6,4}-$ константы, и $\mathscr{D}_{P_{4}, 5}(h)=$ $\mathscr{D}_{5}(h)-c_{6,2} \mathscr{D}_{1}(h)^{3}-c_{6,3} \mathscr{D}_{2}(h)^{2}-c_{6,4} \mathscr{D}_{1}(h) \mathscr{D}_{3}(h)$.

Система (4.7) имеет вид

$$
\begin{gathered}
\frac{d}{d t} r=-\left(\delta+\frac{1}{2}\right) h, \quad \frac{d}{d t} h=-h^{2}+x_{2}, \quad \frac{d}{d t} x_{2}=x_{3}-4 h x_{2}, \quad \frac{d}{d t} x_{3}=x_{4}-6 h x_{3}, \\
\frac{d}{d t} x_{4}=x_{5}-8 h x_{4}, \quad \frac{d}{d t} x_{5}=c_{6,2} x_{2}^{3}+c_{6,3} x_{3}^{2}+c_{6,4} x_{2} x_{4}-10 h x_{5} .
\end{gathered}
$$

Лемма 8.12. Для $c_{6,2}=0, c_{6,3}=c_{5} u c_{6,4}=c_{5}$ имеем $\mathscr{D}_{P_{4}, 5}(h)=\left(\frac{d}{d t}+\right.$ $10 h) \mathscr{D}_{P_{3}, 4}(h)$.

Следовательно, для $c_{6,2}=0, c_{6,3}=48$ и $c_{6,4}=48$ уравнение $\mathscr{D}_{P_{4}, 5}(h)=0$ получается из уравнения Шази-3 подстановкой $y(t)=-6 h(t)$ и действием оператора $\left(\frac{d}{d t}+10 h\right)\left(\frac{d}{d t}+8 h\right)$.

Лемма 8.13. Для $c_{6,2}=-120, c_{6,3}=24, c_{6,4}=44$ и $y(t)=-10 h(t)$ уравнение $\mathscr{D}_{P_{4}, 5}(h)=0$ принимает вид

$$
y^{\prime \prime \prime \prime \prime}-3 y y^{\prime \prime \prime \prime}+3 y^{2} y^{\prime \prime \prime}-\frac{6}{5} y^{3} y^{\prime \prime}+\frac{9}{50} y^{4} y^{\prime}-\frac{3}{500} y^{6}=0 .
$$

Уравнение (8.11) линейно относительно производных.

Имеем $\operatorname{det} S_{4}(h)=h^{\prime \prime \prime \prime \prime \prime}+6 b h h^{\prime \prime \prime \prime}+15 b\left(h^{\prime}+b h^{2}\right) h^{\prime \prime \prime}+10 b\left(h^{\prime \prime}+6 b h h^{\prime}+2 b^{2} h^{3}\right) h^{\prime \prime}+$ $15 b^{2}\left(\left(h^{\prime}\right)^{2}+3 b h^{2} h^{\prime}+b^{2} h^{4}\right) h^{\prime}+b^{5} h^{6}$. Для $b=5$ получаем $\mathscr{D}_{P_{4}, 5}(h)$ с $c_{6,2}=-45$, $c_{6,3}=-26$ и $c_{6,4}=-31$. 
Следствие 8.14. Функция

$$
\frac{1}{5}\left(\frac{1}{t-a_{1}}+\frac{1}{t-a_{2}}+\frac{1}{t-a_{3}}+\frac{1}{t-a_{4}}+\frac{1}{t-a_{5}}\right)
$$

является общим решением уравнения $\mathscr{D}_{P_{4}, 5}(h)=0$ с $c_{6,2}=-45, c_{6,3}=-26 u$ $c_{6,4}=-31$.

\section{§9. Симметрические квадратичные динамические системы и уравнение теплопроводности}

В этом параграфе мы рассматриваем классическую задачу интегрирования квадратичных динамических систем от $n$ переменных (см. [9]) и исследуем более общий анзац для решений уравнения теплопроводности, такой, что каждое решение симметричной квадратичной динамической системы в общем положении (см. определения 9.1,9.2) определяет решение уравнения теплопроводности в этом анзаце.

9.1. Алгебраически интегрируемые квадратичные динамические системы. Рассмотрим в $\mathbb{C}^{n}$ с координатами $\xi=\left(\xi_{1}, \ldots, \xi_{n}\right)^{\top}$ общую однородную квадратичную динамическую систему

$$
\xi_{k}^{\prime}(t)=\xi^{\top} A_{k} \xi, \quad k=1, \ldots, n,
$$

где $A_{k}$ - постоянные матрицы. Эта система однородна относительно градуировки $\operatorname{deg} t=4, \operatorname{deg} \xi_{i}=-4$. Она определяет линейный оператор $L=L\left(A_{1}, \ldots, A_{n}\right)$ : $\mathbb{C}\left[\xi_{1}, \ldots, \xi_{n}\right] \rightarrow \mathbb{C}\left[\xi_{1}, \ldots, \xi_{n}\right]$

$$
L P\left(\xi_{1}, \ldots, \xi_{n}\right)=\sum_{k} \xi^{\top} A_{k} \xi \frac{\partial}{\xi_{k}} P\left(\xi_{1}, \ldots, \xi_{n}\right) .
$$

Имеем $\operatorname{deg} L=-4$.

Обозначим через $\mathrm{Sym} \subset \mathbb{C}\left[\xi_{1}, \ldots, \xi_{n}\right]$ подкольцо симметричных полиномов.

Определение 9.1. Систему (9.1) мы будем называть симметричной, если $L$ переводит кольцо Sym в себя.

Согласно классическим результатам, Sym - кольцо полиномов от $n$ образующих. Наиболее известные мультипликативные образующие этого кольца элементарные симметрические функции и полиномы Ньютона.

Пусть $a_{1}, \ldots, a_{n}$ - однородный мультипликативный базис кольца Sym c $\operatorname{deg} a_{k}=-4 k$. Тогда из симметричной системы (9.1) получается однородная динамическая система

$$
a_{k}^{\prime}(t)=L a_{k}(t), \quad k=1, \ldots, n .
$$

Из градуировки следует, что эту систему можно переписать в виде

$$
a_{k}^{\prime}(t)=g_{k+1}\left(a_{1}(t), \ldots, a_{k}(t)\right)+c_{k} a_{k+1}(t), \quad k=1, \ldots, n,
$$

где $c_{n}=0$ и $g_{j}\left(a_{1}, \ldots, a_{j-1}\right)$ - однородные полиномы с $\operatorname{deg} g_{j}=-4 j$.

Определение 9.2. Однородная симметричная система (9.1) находится в общем положении, если все $c_{k}$ отличны от $0, k=1, \ldots, n-1$.

Лемма 9.3. Понятие «в общем положении» не зависит от выбора мультипликативного базиса. 
Доказательство. Пусть $a_{1}, \ldots, a_{n}$ и $b_{1}, \ldots, b_{n}$ - два мультипликативных базиса с $\operatorname{deg} a_{j}=\operatorname{deg} b_{j}=-4 j$. Рассмотрим идеал разложимых элементов $J \subset$ Sym. Имеем $a_{k}=C_{k} b_{k} \bmod J$, где $C_{k} \neq 0, C_{k} \in \mathbb{C}$. Таким образом, из $(9.2)$ следует, что

$$
b_{k}^{\prime}(t)=G_{k+1}\left(b_{1}(t), \ldots, b_{k}(t)\right)+c_{k} \frac{C_{k+1}}{C_{k}} b_{k+1}(t), \quad k=1, \ldots, n,
$$

для однородных полиномов $G_{j}\left(b_{1}, \ldots, b_{k}\right)$.

Рассмотрим отображение

$$
S: \mathbb{C}^{n} \rightarrow \mathbb{C}^{n}:\left(\xi_{1}, \ldots, \xi_{n}\right) \mapsto\left(s_{1}\left(\xi_{1}, \ldots, \xi_{n}\right), \ldots, s_{n}\left(\xi_{1}, \ldots, \xi_{n}\right)\right),
$$

где $s_{k}$ есть $k$-й элементарный симметрический полином от $\xi_{1}, \ldots, \xi_{n}$. Индуцированное отображение $S^{*}$ переводит полиномы от $s_{1}, \ldots, s_{n}$ в симметрические полиномы от $\xi_{1}, \ldots, \xi_{n}$, более того, каждый симметрический полином от $\xi_{1}, \ldots, \xi_{n}$ есть образ некоторого полинома от $s_{1}, \ldots, s_{n}$. Наш подход к интегрированию квадратичных динамических систем использует соотношение

$$
\xi_{j}^{n}-s_{1} \xi_{j}^{n-1}+\cdots+(-1)^{n} s_{n} \equiv 0,
$$

верное для каждого $j$.

Определение 9.4. Назовем систему (9.1) алгебраически интегрируемой набором функиий $h_{1}, \ldots, h_{n}$, если набор корней уравнения

$$
\xi^{n}-h_{1} \xi^{n-1}+\cdots+(-1)^{n} h_{n} \equiv 0
$$

является решением системы (9.1).

Задача 9.5 (алгебраическая интегрируемость). Для заданной системы вида (9.1) найти обыкновенное дифференциальное уравнение от $h$ степени $n$ и дифференциальные полиномы $h_{2}, \ldots, h_{n}$ от $h$, такие, что для каждого решения $h$ полученного уравнения набор $h_{1}=h, h_{2}, \ldots, h_{n}$ алгебраически интегрирует заданную систему.

Теорема 9.6. Для каждой симметричной системы (9.1) в общем положении существует решение задачи алгебрачческой интегрируемости.

Доказательство. Рассмотрим систему (9.2) с $a_{k}=s_{k}$. В условиях теоремы имеем $c_{k} \neq 0$; следовательно, функции $s_{j}(t)$ могут быть выражены как полиномы от $s_{1}(t), \ldots, s_{j-1}(t)$ и их производных из $(j-1)$-го уравнения для $j=2, \ldots, n$. Следовательно, последнее уравнение задает единственное однородное дифференциальное уравнение на $s_{1}(t)$, в котором коэффициент при старшей производной от $s_{1}(t)$ постоянный и ненулевой. Таким образом, набор $\left(h_{1}, \ldots, h_{n}\right)=\left(s_{1}, \ldots, s_{n}\right)$ алгебраически интегрирует систему $(9.2)$.

Пример 9.7. Классическая система Дарбу-Альфана

$$
\xi_{1}^{\prime}=\xi_{2} \xi_{3}-\xi_{1} \xi_{2}-\xi_{1} \xi_{3}, \quad \xi_{2}^{\prime}=\xi_{1} \xi_{3}-\xi_{1} \xi_{2}-\xi_{2} \xi_{3}, \quad \xi_{3}^{\prime}=\xi_{1} \xi_{2}-\xi_{1} \xi_{3}-\xi_{2} \xi_{3}
$$

алгебраически интегрируется набором $\left(h_{1}, h_{2}, h_{3}\right)$, где $-2 h_{1}-$ решение уравнения Шази-3 (см. (8.3)), $h_{2}=-h_{1}^{\prime}$ и $h_{3}=-\frac{1}{6} h_{2}^{\prime}=\frac{1}{6} h_{1}^{\prime \prime}$.

Пример 9.8. Общая двумерная квадратичная симметричная динамическая система имеет вид

$$
\xi_{1}^{\prime}=\alpha \xi_{1}^{2}+\beta \xi_{1} \xi_{2}+\gamma \xi_{2}^{2}, \quad \xi_{2}^{\prime}=\gamma \xi_{1}^{2}+\beta \xi_{1} \xi_{2}+\alpha \xi_{2}^{2} .
$$


Функция $h_{1}=\xi_{1}+\xi_{2}+\xi_{3}$ является решением дифференциального уравнения $h^{\prime \prime}+\lambda_{1} h^{\prime} h+\lambda_{2} h^{3}=0$, где $\lambda_{1}=(\gamma-3 \alpha-\beta), \lambda_{2}=(\alpha-\gamma)(\alpha+\beta+\gamma)$. При $\beta \neq \alpha+\gamma$ система (9.4) находится в общем положении и

$$
h_{2}=\frac{1}{2(\beta-\alpha-\gamma)} h_{1}^{\prime}-\frac{\alpha+\gamma}{2(\beta-\alpha-\gamma)} h_{1}^{2} .
$$

Пример 9.9. Общая трехмерная квадратичная симметричная динамическая система имеет вид

$$
\begin{aligned}
& \xi_{1}^{\prime}=\alpha \xi_{2} \xi_{3}+\beta \xi_{1}\left(\xi_{2}+\xi_{3}\right)+\gamma \xi_{1}^{2}+\delta\left(\xi_{2}^{2}+\xi_{3}^{2}\right), \\
& \xi_{2}^{\prime}=\alpha \xi_{1} \xi_{3}+\beta \xi_{2}\left(\xi_{1}+\xi_{3}\right)+\gamma \xi_{2}^{2}+\delta\left(\xi_{1}^{2}+\xi_{3}^{2}\right), \\
& \xi_{3}^{\prime}=\alpha \xi_{1} \xi_{2}+\beta \xi_{3}\left(\xi_{1}+\xi_{2}\right)+\gamma \xi_{3}^{2}+\delta\left(\xi_{1}^{2}+\xi_{2}^{2}\right) .
\end{aligned}
$$

Функция $h_{1}=\xi_{1}+\xi_{2}+\xi_{3}$ является решением дифференциального уравнения

$$
\lambda_{1} h^{\prime \prime \prime}+\lambda_{2} h^{\prime \prime} h+\lambda_{3}\left(h^{\prime}\right)^{2}+\lambda_{4} h^{\prime} h^{2}+\lambda_{5} h^{4}=0,
$$

где

$$
\begin{aligned}
\lambda_{1}= & \alpha+2 \beta-2 \gamma-4 \delta, \\
\lambda_{2}= & (\alpha-3 \beta-4 \gamma+2 \delta)(\alpha+2 \beta-2 \gamma-4 \delta), \\
\lambda_{3}= & 2\left(\alpha^{2}-3 \alpha \beta-\beta^{2}+\alpha \gamma-2 \beta \gamma+3 \gamma^{2}-2 \alpha \delta+6 \beta \delta+2 \gamma \delta+\delta^{2}\right), \\
\lambda_{4}=2\left(-\alpha^{3}-2 \alpha^{2} \beta+\alpha \beta^{2}+2 \beta^{3}-2 \alpha^{2} \gamma+6 \alpha \beta \gamma+8 \beta^{2} \gamma+2 \alpha \gamma^{2}-4 \beta \gamma^{2}-6 \gamma^{3}\right. & \\
& \left.\quad+4 \alpha \beta \delta+4 \alpha \gamma \delta-12 \beta \gamma \delta-8 \gamma^{2} \delta+6 \alpha \delta^{2}-16 \beta^{2} \delta+4 \beta \delta^{2}+10 \gamma \delta^{2}+4 \delta^{3}\right), \\
\lambda_{5}=2(\alpha+2 \beta+\gamma+2 \delta)\left(\gamma \alpha^{2}-\gamma \beta^{2}-\alpha \gamma^{2}+\gamma^{3}-2 \alpha \beta \delta+2 \beta^{2} \delta+2 \beta \gamma \delta\right. & \\
& \left.+\alpha \delta^{2}-2 \beta \delta^{2}-3 \gamma \delta^{2}+2 \delta^{3}\right) .
\end{aligned}
$$

При $\alpha+2 \beta-2 \gamma-4 \delta \neq 0, \alpha-\beta+\gamma-\delta \neq 0$ система находится в общем положении и

$$
\begin{gathered}
(\alpha+2 \beta-2 \gamma-4 \delta) h_{2}=h_{1}^{\prime}-(\gamma+2 \delta) h_{1}^{2} \\
3(\alpha-\beta+\gamma-\delta)(\alpha+2 \beta-2 \gamma-4 \delta) h_{3}=-\left(\alpha \gamma+\beta \gamma+\gamma^{2}-2 \beta \delta+\gamma \delta-2 \delta^{2}\right) h_{1}^{3} \\
+(\alpha+\beta+3 \gamma-\delta) h_{1}^{\prime} h_{1}-h_{1}^{\prime \prime} .
\end{gathered}
$$

Пример 9.10. Система

$$
\begin{aligned}
& \xi_{1}^{\prime}=\xi_{2} \xi_{3}-\xi_{1}\left(\xi_{2}+\xi_{3}\right)+\tau^{2}, \\
& \xi_{2}^{\prime}=\xi_{1} \xi_{3}-\xi_{2}\left(\xi_{1}+\xi_{3}\right)+\tau^{2}, \\
& \xi_{3}^{\prime}=\xi_{1} \xi_{2}-\xi_{3}\left(\xi_{1}+\xi_{2}\right)+\tau^{2},
\end{aligned}
$$

где $\tau^{2}=a^{2}\left(\xi_{1}-\xi_{2}\right)\left(\xi_{3}-\xi_{1}\right)+b^{2}\left(\xi_{2}-\xi_{3}\right)\left(\xi_{1}-\xi_{2}\right)+c^{2}\left(\xi_{3}-\xi_{1}\right)\left(\xi_{2}-\xi_{3}\right)$, была впервые изучена Альфаном как естественное обобщение классической системы Дарбу-Альфана (см. [10]). Эта система является симметричной при $a=b=c$ и соответствует параметрам $\alpha=a^{2}+1, \beta=a^{2}-1, \gamma=-a^{2}, \delta=-a^{2}$. В этом случае функция $y=-2\left(\xi_{1}+\xi_{2}+\xi_{3}\right)$ является решением уравнения (8.3) с $c_{4}=\frac{-24}{9 a^{2}-1}$. 
Определение 9.11. Система (9.1) называется квазисимметричной, если существует невырожденная постоянная матрица $B$, такая, что в координатах $\eta=B \xi$ система является симметричной.

Пример 9.12. Система

$$
\xi_{1}^{\prime}=\gamma_{1} \xi_{2} \xi_{3}, \quad \xi_{2}^{\prime}=\gamma_{2} \xi_{1} \xi_{3}, \quad \xi_{3}^{\prime}=\gamma_{3} \xi_{1} \xi_{2}
$$

квазисимметрична. Она переводится в симметричную систему диагональной матрицей $B=\operatorname{diag}\left(\frac{\gamma_{2} \gamma_{3}}{\gamma_{1}}, \frac{\gamma_{1} \gamma_{3}}{\gamma_{2}}, \frac{\gamma_{1} \gamma_{2}}{\gamma_{3}}\right)$. Система решается в функциях Якоби: $\xi_{1}=$ $\sqrt{\gamma_{1}} \operatorname{sn}(t), \xi_{2}=\sqrt{\gamma_{2}} \operatorname{cn}(t), \xi_{3}=\frac{1}{k} \sqrt{\gamma_{3}} \operatorname{dn}(t)$, где $k$ - параметр эллиптического синуса $\operatorname{sn}(t)$. В симметричном случае имеем $\gamma_{1}=\gamma_{2}=\gamma_{3}$. Положим $\kappa_{1}^{\prime}=\alpha_{1} \frac{\xi_{2} \xi_{3}}{\xi_{1}}$, $\kappa_{2}^{\prime}=\alpha_{2} \frac{\xi_{1} \xi_{3}}{\xi_{2}}, \kappa_{3}^{\prime}=\alpha_{3} \frac{\xi_{1} \xi_{2}}{\xi_{3}}$. Получаем систему

$$
\begin{gathered}
\kappa_{1}^{\prime}=\kappa_{1}\left(\frac{\gamma_{2}}{\alpha_{2}} \kappa_{2}+\frac{\gamma_{3}}{\alpha_{3}} \kappa_{3}-\frac{\gamma_{1}}{\alpha_{1}} \kappa_{1}\right), \quad \kappa_{2}^{\prime}=\kappa_{2}\left(\frac{\gamma_{1}}{\alpha_{1}} \kappa_{1}+\frac{\gamma_{3}}{\alpha_{3}} \kappa_{3}-\frac{\gamma_{2}}{\alpha_{2}} \kappa_{2}\right), \\
\kappa_{3}^{\prime}=\kappa_{3}\left(\frac{\gamma_{1}}{\alpha_{1}} \kappa_{1}+\frac{\gamma_{2}}{\alpha_{2}} \kappa_{2}-\frac{\gamma_{3}}{\alpha_{3}} \kappa_{3}\right) .
\end{gathered}
$$

Для $\gamma_{k}=\alpha_{k}, k=1,2,3$, мы получили трехмерную квадратичную симметричную динамическую систему с $\alpha=0, \beta=1, \gamma=-1, \delta=0$. Таким образом, функция $h_{1}$ является решением дифференциального уравнения $h^{\prime \prime \prime}+h^{\prime \prime} h+2\left(h^{\prime}\right)^{2}-$ $2 h^{\prime} h^{2}=0$ и мы имеем $4 h_{2}=h_{1}^{\prime}+h_{1}^{2}, 24 h_{3}=2 h_{1}^{\prime} h_{1}+h_{1}^{\prime \prime}$. Для $\gamma_{1}=1, \gamma_{2}=-1$, $\gamma_{3}=-k^{2}$ получаем $\xi_{1}=\operatorname{sn}(t), \xi_{2}=\operatorname{cn}(t), \xi_{3}=\operatorname{dn}(t)$. Следовательно, система

$$
\kappa_{k}^{\prime}=\kappa_{k}\left(\sum_{l=1}^{3} \kappa_{l}-2 \kappa_{k}\right), \quad k=1,2,3,
$$

имеет решение $\kappa_{1}=(\ln \operatorname{sn}(t))^{\prime}, \kappa_{2}=(\ln \mathrm{cn}(t))^{\prime}, \kappa_{3}=(\ln \operatorname{dn}(t))^{\prime}$. Результат, что система (9.5) интегрируется в абелевых функциях, был получен С. Ковалевской, см. [9]. Имеем $h_{1}=\kappa_{1}+\kappa_{2}+\kappa_{3}$, и, следовательно, решением уравнения $h^{\prime \prime \prime}+$ $h^{\prime \prime} h+2\left(h^{\prime}\right)^{2}-2 h^{\prime} h^{2}=0$ является эллиптическая функция $\left(\ln \left(\left(\operatorname{sn}(t)^{2}\right)^{\prime}\right)\right)^{\prime}$.

Пример 9.13. В примере 9.9 для $\alpha=0, \beta=0, \gamma=-3, \delta=0$ функция $h_{1}$ является решением дифференциального уравнения $h^{\prime \prime \prime}+12 h^{\prime \prime} h+9\left(h^{\prime}\right)^{2}+$ $54 h^{\prime} h^{2}+27 h^{4}=0$, совпадающего с (8.2) для $c_{4}=-3$.

Положим $m_{i}=\xi_{i}-\frac{1}{3}\left(\xi_{1}+\xi_{2}+\xi_{3}\right)$. Тогда $s_{1}\left(m_{i}\right)=0$.

Следствие 9.14. Функиия $\psi(z, t)$, такая, что $\psi(z, t)=e^{-\frac{1}{2} h(t) z^{2}+r(t)} \sigma(z$; $\left.g_{2}(t), g_{3}(t)\right)$ для некоторых функиий $r(t), h(t), g_{2}(t)$ u $g_{3}(t)$, удовлетворяет уравнению теплопроводности (2.1) тогда и только тогда, когда для некоторого решения $\xi_{1}, \xi_{2}, \xi_{3}$ системь Дарбу-Альфана имеют место формуль

$$
\begin{gathered}
h(t)=\frac{1}{3} s_{1}\left(\xi_{1}, \xi_{2}, \xi_{3}\right), \quad r^{\prime}(t)=-\frac{3}{2} h(t), \\
g_{2}(t)=-4 s_{2}\left(m_{1}, m_{2}, m_{3}\right), \quad g_{3}(t)=4 s_{3}\left(m_{1}, m_{2}, m_{3}\right) .
\end{gathered}
$$

Следствие 9.15. Уравнение эллиптической кривой $y^{2}=4 x^{3}-g_{2} x-g_{3}$ в терминах $m_{i}$ принимает вид $(y / 2)^{2}=\left(x-m_{1}\right)\left(x-m_{2}\right)\left(x-m_{3}\right)$. Таким образом, следствие 9.14 дает решения уравнения теплопроводности в терминах точек ветвления этой кривой. 


\section{2. Решения уравнения теплопроводности в терминах квадратич-} ных динамических систем. Мы исследовали в деталях анзац (3.9) для решений уравнения теплопроводности (2.1). Заметим, что анзац (3.9) является частным случаем анзаца

$$
\psi(z, t)=e^{r(t)} \Psi(z ; \boldsymbol{x}(t)),
$$

где

$$
\Psi(z ; \boldsymbol{x})=z^{\delta}+\sum_{k \geqslant 1} \Psi_{k}(\boldsymbol{x}) \frac{z^{2 k+\delta}}{(2 k+\delta) !}
$$

$\operatorname{deg} z=2, \boldsymbol{x}=\left(x_{1}, x_{2}, \ldots, x_{n+1}\right), \operatorname{deg} x_{q}=-4 q$ и $\Psi_{k}(\boldsymbol{x})$ - однородные полиномы степени $-4 k$.

Используя подход, развитый в $\S \S 3$ и 4, мы получаем следующие результаты:

Теорема 9.16. Из любых двух из трех следующих условий вытекает треmbe:

1) функиия $\psi(z, t)$ вида (9.6) удовлетворяет уравнению теплопроводности $(2.1)$;

2) коэфбиииенты функиии $\Psi(z ; \boldsymbol{x})$ задаются соотношением

$$
\Psi_{k+1}(\boldsymbol{x})=2 \sum_{j=1}^{n+1} p_{j+1}(\boldsymbol{x}) \frac{\partial}{\partial x_{j}} \Psi_{k}(\boldsymbol{x})+\Psi_{1}(\boldsymbol{x}) \Psi_{k}(\boldsymbol{x})
$$

с начальными условиями $r^{\prime}(t)=\frac{1}{2} \Psi_{1}(\boldsymbol{x}(t))$, где $p_{k}(\boldsymbol{x})$ - однородные полиномы;

3) набор функиий $x_{k}(t)$ является решением однородной полиномиальной динамической системь

$$
\frac{d}{d t} x_{k}=p_{k+1}(\boldsymbol{x}), \quad k=1, \ldots, n+1 .
$$

Пример 9.17. Для $n=2$ положим $x_{1}(t)=\frac{1}{3}\left(\frac{1}{t-a_{1}}+\frac{1}{t-a_{2}}+\frac{1}{t-a_{3}}\right)$. Тогда для $x_{2}(t)=-\frac{1}{3}\left(\frac{1}{\left(t-a_{1}\right)^{2}}+\frac{1}{\left(t-a_{2}\right)^{2}}+\frac{1}{\left(t-a_{3}\right)^{2}}\right)$ и $x_{3}(t)=\frac{2}{3}\left(\frac{1}{\left(t-a_{1}\right)^{3}}+\frac{1}{\left(t-a_{2}\right)^{3}}+\frac{1}{\left(t-a_{3}\right)^{3}}\right)$ имеем

$$
\frac{d}{d t} x_{1}=x_{2}, \quad \frac{d}{d t} x_{2}=x_{3}, \quad \frac{d}{d t} x_{3}=-3\left(4 x_{1} x_{3}+3 x_{2}^{2}+18 x_{2} x_{1}^{2}+9 x_{1}^{4}\right) .
$$

Положим $\mathscr{L}=2 x_{2} \frac{\partial}{\partial x_{1}}+2 x_{3} \frac{\partial}{\partial x_{2}}-6\left(4 x_{1} x_{3}+3 x_{2}^{2}+18 x_{2} x_{1}^{2}+9 x_{1}^{4}\right) \frac{\partial}{\partial x_{3}}+\Psi_{1}(\boldsymbol{x})$.

Из теоремы 9.16 следует, что для функции $\Psi(z ; \boldsymbol{x})$ вида $(9.7)$, где $\Psi_{1}(\boldsymbol{x})=$ $-\frac{1}{2} x_{1}$ и $\Psi_{k}(\boldsymbol{x})=\mathscr{L}^{k-1} \Psi_{1}(\boldsymbol{x})$, функция

$$
\psi(z, t)=\frac{1}{\sqrt[12]{\left(t-a_{1}\right)\left(t-a_{2}\right)\left(t-a_{3}\right)}} \Psi(z ; \boldsymbol{x}(t))
$$

удовлетворяет уравнению теплопроводности.

Используя методы 4 , мы приводим систему (9.8) к виду

$$
\frac{d}{d t} x_{k}=x_{k+1}, \quad k=1, \ldots, n, \quad \frac{d}{d t} x_{n+1}=K_{n}(\boldsymbol{x}) .
$$

Теорема 9.18. Каждое решение уравнения теплопроводности вида (9.6) задается набором $\left(n, K_{n}, x_{1}, r_{0}\right)$, где $n$ - натуральное число, $K_{n}$ - однородный полином $K_{n}(\boldsymbol{x})$ градуировки $-4(n+2), x_{1}$ - решение $x_{1}(t)$ уравнения $x_{1}^{(n+1)}=$ $K_{n}\left(x_{1}, x_{1}^{\prime}, \ldots, x_{1}^{(n)}\right), r_{0}-$ константа. 
Следствие 9.19. Каждое решение уравнения теплопроводности вида (9.6) определяется конечномерным числовым вектором $\left(n, \boldsymbol{K}_{n}, \boldsymbol{C}_{n}, r_{0}\right)$, где $\boldsymbol{K}_{n}-$ вектор коэфбициентов полинома $K_{n}(\boldsymbol{x})$ и $\boldsymbol{C}_{n}$ - вектор началъных данных задачи Кочи уравнения $x_{1}^{(n+1)}=K_{n}\left(x_{1}, x_{1}^{\prime}, \ldots, x_{1}^{(n)}\right)$.

Из теорем 9.6 и 9.16 получаем

Следствие 9.20. Каждая симметричная квадратичная динамическая система в общем положении определяет решение уравнения теплопроводности вида (9.6).

\section{ЛитерАтУРА}

[1] Е. Ю. Бунькова, В. М. Бухштабер, Уравнения теплопроводности и семейства двумерных сигма-функиий, в кн.: Геометрия, топология и математическая физика. II, Сб. статей. K 70-летию со дня рождения академика С. П. Новикова, Труды МИАН, т. 266, МАИК, М., 2009, 5-32.

[2] П. Олвер, Приложения групп Ли к дифференииалъным уравнениям, Мир, М., 1989.

[3] Р. Конт, М. Мюзетт, Метод Пенлеве и его приложения, РХД, Москва-Ижевск, 2011.

[4] В. М. Бухштабер, Д. В. Лейкин, М. В. Павлов, Егоровские гидродинамические цепочки, уравнение Шази и группа $S L(2, \mathbb{C})$, Функц. анализ и его прил., 37:4 (2003), 13-26.

[5] S. Chakravarty, M. J. Ablowitz, P. A. Clarkson, Reductions of self-dual Yang-Mills fields and classical systems, Phys. Rev. Lett., 65:9 (1990), 1085-1087.

[6] B. Dubrovin, Geometry of 2D topological field theories, in: Lecture Notes in Math., vol. 1620, Springer-Verlag, Berlin, 1996, 120-348.

[7] P. A. Clarkson, P. J. Olver, Symmetry and the Chazy equation, J. Differential Equations, 124:1 (1996), 225-246.

[8] Э. Т. Уиттекер, Дж. Н. Ватсон, Курс современного анализа, ч. 2. Трансцендентные функиии, УРСС, М., 2010.

[9] Переписка С. В. Ковалевской и Г. Миттаг-Леффлера, в кн.: Научное наследство, т. 7, Наука, М., 1984, Письмо 57.

[10] S. Chakravarty, R. G. Halburd, First integrals and gradient flow for a generalized Darboux-Halphen system, in: Contemp. Math., vol. 301, Amer. Math. Soc., Providence, RI, 2002, 273-281.

[11] В. М. Бухштабер, Д. В. Лейкин, Законы сложения на якобианах плоских алгебрачческих кривых, в кн.: Труды МИАН, т. 251, Наука, М., 2005, 54-126.

Математический институт им. В. А. Стеклова РАН e-mail: bunkova@mi.ras.ru

Поступило в редакцию

Математический институт им. В. А. Стеклова РАН e-mail: buchstab@mi.ras.ru 